\title{
Der Ausbau der deutschen Stromnetze: Kohärente Parteienideologie oder Sollbruchstelle entlang lokaler Interessen?
}

\author{
Simon Fink $\cdot$ Hendrik Teichgräber $\cdot$ Mareike Wehling
}

Eingegangen: 4. Januar 2021 / Überarbeitet: 28. Juli 2021 / Angenommen: 27. Oktober 2021 / Online publiziert: 24. November 2021

(C) Der/die Autor(en) 2021

Zusammenfassung Der Ausbau der Stromtrassen ist ein zentrales Element der deutschen Energiewende. Die Forschung weiß aber noch wenig darüber, inwieweit dieses Politikfeld durch Parteiendifferenz strukturiert wird. Insbesondere über die lokale Ebene gibt es noch wenig Forschung. Der Beitrag formuliert zwei Hypothesen. Der ersten Hypothese zufolge sind lokale Parteipositionen zum Stromnetzausbau die selben Positionen wie die Positionen der Landesverbände. Die zweite Hypothese vermutet, dass durch unterschiedliche Betroffenheit lokale parteipolitische Akteure von den Positionen ihrer Landesverbände abweichen. Ein Vergleich von zwölf niedersächsischen Landkreisen unterstützt Hypothese 2. Parteipolitische Akteure in Landkreisen, die vom Stromexport profitieren, betonen systematisch die positiven Aspekte des Stromnetzausbaus und verschweigen kritische Aspekte. Bei Akteuren in reinen Transit-Landkreisen ist es umgekehrt. Der Beitrag zeigt daher, dass Parteiendifferenz in der Umweltpolitik in hohem Maße kontextabhängig ist. Auf lokaler Ebene - die für die Umsetzung von Umweltpolitik bedeutsam ist - können Parteipräferenzen systematisch von den Präferenzen höherer Ebenen abweichen.

Schlüsselwörter Parteiendifferenz · Lokale Politik · Umweltpolitik · Parteipräferenzen

\footnotetext{
Prof. Dr. Simon Fink $(\bowtie) \cdot$ Hendrik Teichgräber $\cdot$ Mareike Wehling Institut für Politikwissenschaft, Georg-August-Universität Göttingen, Platz der Göttinger Sieben 3, 37073 Göttingen, Deutschland

E-Mail: simon.fink@sowi.uni-goettingen.de

Hendrik Teichgräber

E-Mail: h.teichgraeber@stud.uni-goettingen.de

Mareike Wehling

E-Mail: mareike.wehling@stud.uni-goettingen.de
} 


\title{
Electricity grid construction in Germany: Coherent partisan ideology or cleavage along local economic interests?
}

\begin{abstract}
The construction of new transmission grids for electricity is central for the German Energiewende. However, we know little about partisan differences in this policy field. Particularly the local level is under-researched. This article develops two hypotheses: According to the first, local parties have the same preferences as their federal state parties. According to the second, local parties diverge from the positions of their "higher" party organizations, based on their economic situation. An analysis of twelve districts in Lower Saxony supports hypothesis 2. Parties in districts that profit from exporting electricity emphasize the benefits of electricity grids. Conversely, parties in mere transit districts emphasize the downsides of electricity grid construction. The article thus shows that partisan differences in environmental policy are context-dependent. On the local level—-that may be important for the actual implementation of policies-party preferences may systematically diverge from of higher-level party preferences.
\end{abstract}

Keywords Partisan differences $\cdot$ Local politics $\cdot$ Environmental policy $\cdot$ Party preferences

\section{Einleitung}

Die Energiewende im Bereich Strom ist ein zentrales Projekt der deutschen Politik an der Schnittstelle von Umwelt- und Wirtschaftspolitik (Radtke und Kersting 2018). Um die Transformation zu erneuerbaren Energien zu bewältigen, ist der Bau zahlreicher Stromtrassen geplant.

An der Planung der Stromtrassen sind alle Ebenen des deutschen Mehrebenensystems beteiligt: Die Bedarfsplanung liegt beim Bund. Raumordnung und Planfeststellung erfolgen je nach Bedeutung der Trasse entweder durch die Bundesnetzagentur oder die Bundesländer (Steinbach 2013). Letztlich gebaut werden die Trassen auf dem Gebiet von Kommunen.

Die Forschung zeigt, dass sich die Parteien auf Bundesebene relativ einig über den Ausbau der Stromnetze sind, dass aber ihre Landesverbände ihre Präferenzen von regionalen ökonomischen Gegebenheiten leiten lassen (Fink et al. 2019). Parteien aus Bundesländern, die vom Stromexport profitieren, sind für den Netzausbau, Parteien aus Transit-Bundesländern sind dagegen - unabhängig von der Position der Bundespartei.

Die bisherige Forschung lässt die unterste Ebene des deutschen Mehrebenensystems außer Acht. Kommunalpolitik galt lange Zeit als wenig von Parteipolitik geprägt (Holtmann et al. 2017, S. 124-144). Diese Sichtweise ist aber weitgehend widerlegt. Zahlreiche Studien zeigen, dass lokale Parteien unterschiedliche Präferenzen haben, und sich diese Präferenzen auf Policies auswirken (Debus et al. 2012; Gross und Jankowski 2020a). Für das vorliegende Politikfeld wissen wir zwar, dass lokale Parteien in den Konflikten um Stromtrassen eine zentrale Rolle spielen (Mes- 
singer-Zimmer und Zilles 2016), aber noch wenig darüber, wie sich ihre Präferenzen erklären lassen.

An dieser Forschungslücke setzt dieser Beitrag an. Er untersucht, ob sich die Präferenzen deutscher Parteien auf lokaler Ebene durch ideologische Homogenität mit dem Landesverband oder durch lokale ökonomische Gegebenheiten erklären lassen. Die erste Hypothese folgt dem Argument der ,kurzen Leine“ (Müller 2013). Diesem Argument zufolge haben Parteien ein Interesse an einem homogenen ideologischen Bild nach außen. Die zweite Hypothese vermutet, dass Parteien Anreize haben, ihre Programmatik an lokale Gegebenheiten anzupassen, um Wähler*innen anzuziehen (Bräuninger und Debus 2012). Beide Hypothesen wurden bisher anhand der Beziehung von Bundesparteien zu ihren Landesverbänden getestet, sind aber analog auf andere Ebenen anwendbar.

Der Beitrag testet beide Hypothesen anhand einer Untersuchung niedersächsischer Kreisverbände und Landtagsabgeordneter. Niedersachsen ist quasi eine Bundesrepublik im Kleinen. Die Regionen im Norden, die vom Ausbau der Windkraft profitieren, haben ein Interesse am Ausbau der Stromnetze, um Windstrom verkaufen zu können; die Regionen im Süden tragen die politischen Kosten der bei den Bürger*innen unbeliebten Stromleitungen, ohne den ökonomischen Nutzen zu haben. Daher vergleicht dieser Artikel die Positionen von Kreisverbänden und Landtagsabgeordneten von CDU, SPD und Grünen aus nord- und südniedersächsischen Kreisen. Die Frage ist, ob die lokalen Akteure ihren Landesverbänden folgen (Hypothese 1), oder ob die Akteure in Südniedersachsen kritischer gegenüber dem Stromnetzausbau sind (Hypothese 2).

Das Ergebnis ist, dass sich eher Hypothese 2 bestätigt. Zwar finden sich keine Kreisverbände und kein Mitglied des Landtages, die der offiziellen Linie der Landtagswahlprogramme widersprechen. Allerdings findet sich ein systematisches Muster der Betonung von Positionen. Die Landesverbände haben in ihren Programmen meist abwägende Positionen zum Ausbau der Stromnetze - grundsätzlich befürwortend, aber mit Einschränkungen und unter Bedingungen. Die lokalen Akteure heben selektiv entweder die Befürwortung des Netzausbaus oder die Einschränkungen und Bedingungen hervor, je nach dem, ob sie in Nord- oder Südniedersachsen angesiedelt sind. Es zeigt sich also keine Parteiendifferenz, sondern eine Dominanz der regionalen Kontexteffekte (Fink et al. 2019).

Damit trägt unser Beitrag zu drei Debatten bei. Erstens zeigt unser Ergebnis, dass der Ausbau der deutschen Stromnetze schwer zu koordinieren ist (Benz 2019; Ohlhorst et al. 2013). Die übliche Vermutung ist, dass Parteien Koordinierung über die Ebenen hinweg leisten - z. B. dass „die SPD“ über alle Ebenen des Mehrebenensystems hinweg die selben Präferenzen hat. Dies ist aber nicht der Fall. Es ist möglich, dass eine Partei im Bund, in einem Bundesland und in einem Kreis die Mehrheit hat, und es dennoch zwischen diesen drei Ebenen zu Konflikten über Stromleitungen kommt. Zweitens zeigt unser Ergebnis ein Politikfeld, in dem die deutschen Parteien nur wenig hierarchisch funktionieren, sondern es Bruchlinien zwischen den Ebenen gibt (Detterbeck und Renzsch 2003; Stecker 2015). Eine offene Frage ist, inwieweit dies an den Spezifika des Politikfeldes liegt - insbesondere der klaren geographischen Zurechenbarkeit von Kosten und Nutzen der Policy (siehe auch Lindloff et al. 2017). Drittens mahnt unser Beitrag für die Frage der Parteiendifferenz in der Um- 
weltpolitik dazu, die unteren Ebenen im deutschen Mehrebenensystem in den Fokus zu nehmen (Töller 2017). Allein die Tatsache, dass eine Parteiendifferenz auf Bundesebene besteht, bedeutet nicht, dass diese Differenz auch auf den unteren Ebenen des Mehrebenensystems besteht. Das ist wichtig, wenn die Verantwortung für die Implementation von Umweltpolitiken - die letztlich für die Wirkung der Policies zentral ist - bei den unteren Ebenen liegt.

Der Artikel gliedert sich in fünf Abschnitte. Nach der Einleitung schildert der zweite Abschnitt das Politikfeld Stromnetzausbau. Abschnitt 3 diskutiert das theoretische Argument. Abschnitt 4 beschreibt das Forschungsdesign und die Datenbasis. Abschnitt 5 enthält die empirische Analyse, Abschnitt 6 fasst zusammen.

\section{Das Politikfeld: Der Ausbau der deutschen Stromnetze}

Der Ausbau der deutschen Stromnetze ist eine Policy an der Schnittstelle von Energie-, Umwelt- und Wirtschaftspolitik.

Er ist Teil der Energiepolitik, da sein Ziel ist, die Energiewende hin zu erneuerbaren Energien zu ermöglichen. Die politischen Weichenstellungen der Energiewende begünstigen eine Strategie, die darauf beruht, den deutschen Strombedarf durch an der Küste produzierten Windstrom zu decken (Funcke und Bauknecht 2016). Um diesen Windstrom in die industriellen Zentren in Süddeutschland zu transportieren, ist ein Ausbau der Übertragungsnetze notwendig (Steinbach 2013).

Er betrifft aber auch die Umweltpolitik. Einerseits - dies ist die Position vieler bundespolitischer Akteure - ist der Ausbau der Stromnetze notwendig, um den Übergang zu erneuerbaren Energien zu bewältigen, und daher umweltpolitisch positiv. Andererseits - diese Position wird stärker, je näher die Akteure an den konkreten Stromleitungen sind - sind Stromleitungen große Infrastrukturen mit negativen Folgen für die Umwelt, beispielsweise dem großräumigen Zerschneiden von Lebensräumen.

Last but not least hat der Stromnetzausbau wirtschaftspolitische Implikationen. Je nach dem, in welcher Form er vorangeht, begünstigt er einige wirtschaftliche Akteure und benachteiligt andere (beispielsweise die Eigentümer*innen von Windparks; Landwirt*innen, über deren Land die Trassen verlaufen; Tourismusregionen, die Einbußen befürchten etc.).

In diesem Spannungsfeld bewegen sich die Parteien. Sie müssen sich zum Ausbau der Stromnetze positionieren. Dies bezieht sich sowohl auf die ,große“ Frage, ob man den Ausbau der Stromnetze befürwortet, als auch auf Fragen der Ausgestaltung: Befürwortet man die (teuren) Erdkabel? Welche Trasse wird gewählt? Will man sie durch Gegenden führen, die wenig Infrastruktur haben, oder sie mit bestehenden Infrastrukturen bündeln? Welche energie-, umwelt- und wirtschaftspolitischen Implikationen haben die Entscheidungen? Und für Akteure mit Wiederwahlmotivation entscheidend: Wie reagieren die Wähler*innen auf die Vorschläge?

Aus der Komplexität dieser Abwägungen ergibt sich, dass sich die Positionen der Parteien zum Ausbau der Stromnetze kaum a priori bestimmen lassen. Die ökonomische links-rechts-Achse des Parteienwettbewerbs ist für die Umweltpolitik nur begrenzt aussagekräftig (Carter 2013); aber auch die für die Umweltpolitik übli- 
cherweise aussagekräftigere Materialismus-Postmaterialismus-Dimension ist wenig hilfreich, da nicht klar ist, ob die Umweltauswirkungen des Stromnetzausbaus positiv sind (als Teil der Energiewende), oder die Kosten für die Umwelt als zu hoch erachtet werden (als großes Infrastrukturprojekt).

Eine Position zum Stromnetzausbau müssen die deutschen Parteien auf allen Ebenen finden. Auf der Bundesebene hat der Gesetzgeber mit dem Energieleitungsausbaugesetz (EnLAG), dem Energiewirtschaftsgesetz (EnWG) und dem Netzausbaubeschleunigungsgesetz (NABEG) die grundlegenden Rechtsakte erlassen. Die Bundesebene - hier vor allem die Bundesnetzagentur - koordiniert die Bedarfsplanung für die Stromnetze. Außerdem führt die Bundesnetzagentur für bundesweit bedeutsame Trassen das Raumplanungs- und Planfeststellungsverfahren durch, konkretisiert also den Verlauf der Stromtrassen bis zum Bau (Steinbach 2013).

Die Bundesländer sind ebenso wichtig wie die Bundesebene. Sie liefern die Daten zum Ausbau erneuerbarer Energien, auf deren Basis die Bedarfsfeststellung durchgeführt wird (Fink und Koch 2016). Außerdem sind sie für die Raumplanung und Planfeststellung vieler Trassen zuständig und können Entscheidungen über Verlauf und Ausführung treffen (Steinbach 2013).

Die Landkreise haben keine formale Rolle beim Bau der Stromnetze, ihre informelle Rolle ist aber bedeutsam. Zunächst können sie an den Beteiligungsverfahren auf den oberen Ebenen teilnehmen und ihre Expertise einspeisen (Fink und Ruffing 2018, 2019). Außerdem sind kommunale Akteure zentral für lokale Proteste (Messinger-Zimmer und Zilles 2016; Vogeler und Bandelow 2016). Zwar sind hier auch Bürgerinitiativen wichtige Akteure, aber wie sich die lokale Politik positioniert, kann für den Erfolg von Bürgerinitiativen entscheidend sein (Reusswig et al. 2016).

Zusammenfassend ist der Ausbau der deutschen Stromnetze ein Politikfeld, das parteipolitische Akteure auf allen Ebenen des deutschen Systems zwingt, sich zu positionieren. Der nächste Abschnitt entwickelt zwei theoretische Argumente darüber, wie diese Positionierung geschehen kann.

\section{Das theoretische Argument: Einheitliche Parteipräferenzen im Mehrebenensystem oder Dominanz lokaler Interessen?}

Die Präferenzen der lokalen Parteien im deutschen System sind lange Zeit nicht beforscht worden. Dies liegt zum Teil an der These, auf lokaler Ebene gebe es kaum Parteipolitik (Holtmann et al. 2017, S. 124), zum Teil an der schlechten Datenverfügbarkeit. Beide Hindernisse sind aber im Schwinden begriffen. Auf der einen Seite zeigen immer mehr Studien, dass auf lokaler Ebene programmatischer Parteienwettbewerb stattfindet (Holtkamp et al. 2015; Kersting und Schneider 2016), und dass die Präferenzen lokaler Parteien einen Unterschied für Koalitionen oder Policies machen (Debus et al. 2012; Schammann 2015). Auf der anderen Seite bietet das Local Manifestos Projekt einen Datenkorpus, der für deutsche Großstädte Volltexte der Wahlprogramme bereitstellt (Gross und Jankowski 2020a, b).

Der vorliegende Beitrag beforscht die Frage der Parteiendifferenz nicht direkt, sondern transformiert sie in eine Frage der vertikalen Übereinstimmung von Parteipositionen im Mehrebenensystem. Für uns ist die Frage zentral, wie die Position 
lokaler deutscher Parteien und parteipolitischer Akteure im Vergleich zu ihren Landesverbänden ist. Diese Frage ist analog zur Frage, wie groß der Spielraum der Landesverbände in Relation zu ihren Bundesparteien ist. Hier gibt es zwei Thesen.

Die erste Hypothese betrachtet Parteien als Top-down-Organisationen und sieht „niedrigere“ Parteiuntergliederungen als Agenten der darüberliegenden Parteiebene (Müller 2013). Das Argument ist, dass in Mehrebenensystemen wesentliche Aufgaben von Bund, Ländern und Gemeinden erfüllt werden, und es für Wähler*innen nicht klar ersichtlich ist, wer agiert (Däubler et al. 2018). Für das deutsche System trifft dieses Argument zu: Für Wähler*innen ist nicht klar erkennbar, ob die Gemeinde oder der Landkreis ihnen als selbst handelnde Akteure gegenübertreten, oder sie nur im Auftrag des Bundes oder des Landes handeln. Die Zuschreibung von politischer Verantwortung ist damit erschwert. Wähler*innen könnten Parteien bei Landtagswahlen für Policies bestrafen, die auf lokaler Ebene entstanden sind, und umgekehrt. Für Parteien bedeutet dies, dass sie einen Anreiz haben, einheitliche ideologische Profile zu wahren. Wenn die Positionen einer Partei im Mehrebenensystem zu heterogen werden - wenn die Wähler*innen z. B. keine Übereinstimmung zwischen der lokalen CDU und der CDU auf Landesebene sehen - besteht die Gefahr, dass diese Partei in den Wahlen abgestraft wird (Müller 2013). Somit haben die Landesverbände einen Anreiz, ,ihre“ lokalen Untergliederungen auf Linie zu halten.

Für unsere Untersuchung bedeutet das, dass die Positionen der Landesverbände und die Positionen lokaler Parteien und politischer Akteure zum Netzausbau übereinstimmen sollten. Dies ist sowohl für das kohärente Bild der Parteien, als auch aus Sicht des Politikfeldes sinnvoll: Eine SPD, die auf Landesebene regiert und Stromnetze ausbauen will, hat beispielsweise wenig Interesse daran, dass ihre eigenen lokalen Politiker*innen Teil von Protestkoalitionen gegen Stromtrassen sind.

Hypothese 1 Die Position deutscher lokaler Parteien zum Ausbau der Stromnetze entspricht der Position ihres jeweiligen Landesverbandes.

Die zweite Hypothese sieht Parteien weniger hierarchisch und vermutet, dass es aus Gründen des vote seeking rational sein kann, sich an lokale Wähler*innenpräferenzen anzupassen (Bräuninger und Debus 2012). Dieser Hypothese zufolge sollten homogene Präferenzen zwischen lokalen Parteien und Landesverbänden dann auftreten, wenn die Wähler*innenpräferenzen innerhalb eines Landes homogen sind. Wenn es sich aber aus Wiederwahlgründen lohnt, auf lokale Präferenzen einzugehen (z. B. weil sich die Präferenzen zwischen städtischen und ländlichen Regionen unterscheiden), dann ist es für Parteien rational, den lokalen Parteien Spielraum zu geben, um auf diese Präferenzen zu reagieren.

Für das vorliegende Politikfeld sind solche unterschiedlichen Präferenzen wahrscheinlich. Der Stromnetzausbau betrifft eine erste Gruppe von Landkreisen nicht, da keine Trasse in ihrer Nähe geplant wird (dies ist aus dem Netzentwicklungsplan (ÜNB 2017) abzusehen). Eine zweite Gruppe von Landkreisen hat ein Interesse daran, dass Stromtrassen gebaut werden, da sie zu den Produzenten von Windstrom gehören. Da Strom schlecht gespeichert werden kann, ist es für diese Regionen essenziell, dass Stromtrassen den Strom in die Verbrauchszentren liefern. Jede Ki- 
lowattstunde Strom, die nicht vom Netz aufgenommen werden kann, ist für diese Regionen entgangener monetärer Gewinn. Eine dritte Gruppe von Landkreisen sind die Verlierer des Netzausbaus. Stromtrassen sind im Elektorat unpopulär, weil sie Landschaften zerschneiden, den Wert von Immobilien bedrohen, und als Gesundheitsgefahr gesehen werden (Mueller et al. 2019a). ${ }^{1}$ Dies fällt vor allem dann ins Gewicht, wenn diesen elektoralen Kosten kein ökonomischer Nutzen vor Ort gegenübersteht. Wir könnten also lokal unterschiedliche Präferenzen der Wähler*innen haben, die sich in unterschiedlichen Positionen der Parteien niederschlagen. Je nach dem, wie stark eine Region vom Netzausbau profitiert, sollten sich die lokalen Parteien für oder gegen den Netzausbau positionieren.

Hypothese 2 Die Position deutscher lokaler Parteien zum Ausbau der Stromnetze wird dadurch bestimmt, inwieweit ihre Region vom Stromnetzausbau ökonomisch profitiert.

Diese Hypothese lässt sich in zwei Unterhypothesen spezifizieren. Wie bereits diskutiert, könnte die ökonomische Lage zu zwei Positionen führen: Parteien aus Kreisen, die vom Stromnetzausbau profitieren, sollten sich für den Ausbau positionieren. Parteien aus Kreisen, die nicht profitieren, sollten sich gegen den Ausbau positionieren. ${ }^{2}$

Hypothese 2.1 Parteien aus Kreisen, die vom Stromnetzausbau ökonomisch profitieren, weil sie Windstrom exportieren, sind für den Stromnetzausbau.

Hypothese 2.2 Parteien aus Kreisen, die vom Stromnetzausbau nicht profitieren, sind gegen den Stromnetzausbau.

\subsection{Design und Methode: Vergleich niedersächsischer lokaler Parteien und Repräsentant*innen}

Um die Hypothesen zu testen ist es nötig, die Präferenzen von Parteien zum Stromnetzausbau zu erheben, in einem Vergleich von Regionen, die vom Stromnetzausbau profitieren mit Regionen, die die politischen Kosten des Stromnetzausbaus tragen. Die sinnvolle Einheit für einen solchen Vergleich sind dabei Landkreise. Diese können bereits anhand der Landkarten der Bedarfsplanung (ÜNB 2017) erkennen, ob sie potenziell betroffen sind.

Als ideales Bundesland für einen solchen Vergleich bietet sich Niedersachsen an. Das Flächenland reicht von der Nordseeküste über die norddeutsche Tiefebene bis nach Hessen und Thüringen im Süden. Niedersachsen insgesamt profitiert vom

\footnotetext{
1 Dies korrespondiert mit dem gut bestätigten Befund, dass Befragte zwar meist die Energiewende an sich befürworten, aber lokale Projekte ablehnen (Batel und Devine-Wright 2015; Mueller et al. 2019a, b). Wenn also gerade das lokale Projekt der Outcome des Interesses für Wähler*innen ist, dann sollten sie sich auch für die Position ihrer lokalen Parteien vor Ort interessieren. Empirisch zeigt sich spezifisch in einer Studie zum Netzausbau in Niedersachsen, dass die Menschen an Entscheidungen beteiligt werden wollen, und dass sie Kommunalpolitiker*innen mehr vertrauen als Landespolitiker*innen (Schweizer-Ries 2010).

2 Alternativ könnte es für Parteien aus letzteren Kreisen auch einfach kein salientes Thema sein und sie positionieren sich nicht.
} 


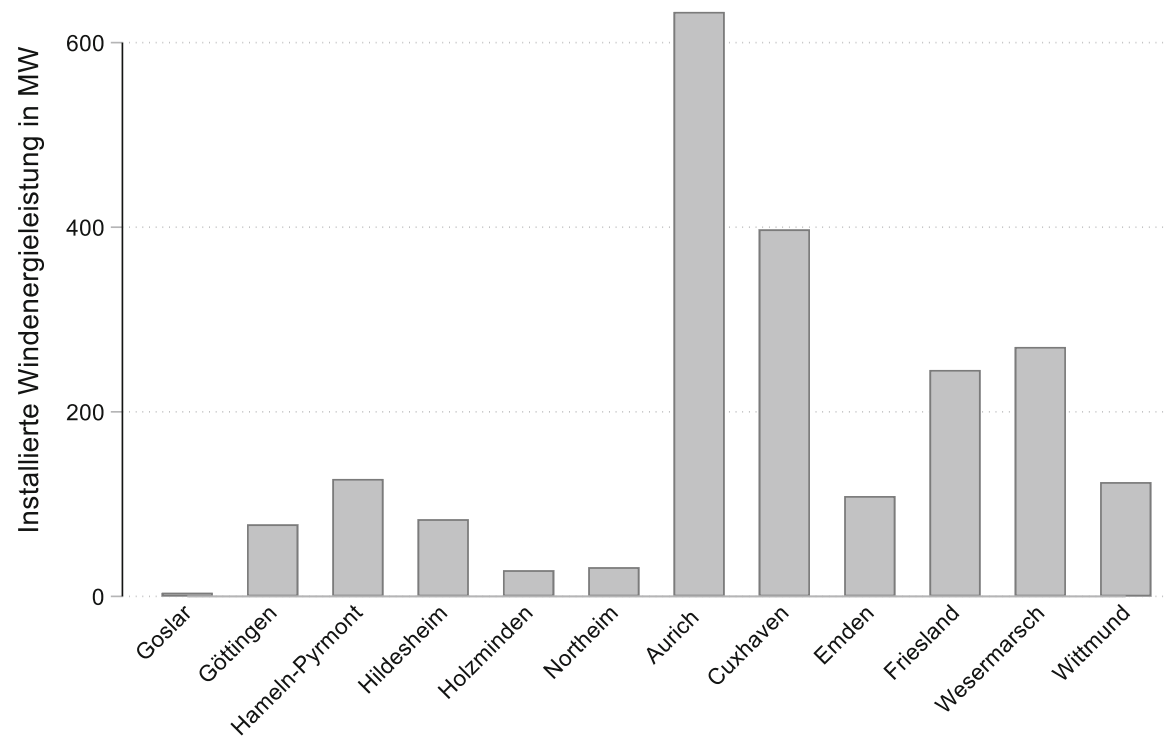

Abb. 1 Installierte Windenergieleistung in MW pro Landkreis. (Quelle Marktstammdatenregister der BNetzA, https://www.marktstammdatenregister.de Stichtag 03.07.2020. Erfasst sind alle Windenergieanlagen auf dem Gebiet des Landkreises. In diesen Daten nicht enthalten sind Offshore-Windparks, die separat in den „Clustern Nordsee“ geführt werden)

Zubau an Windenergie und benötigt Stromnetze, um den Windstrom abzutransportieren. Dementsprechend stehen die niedersächsischen Landeverbände aller Parteien dem Netzausbau positiver gegenüber als die Landesverbände ihrer Schwester-Parteien in anderen Bundesländern (Fink et al. 2019).

Allerdings ist Niedersachsen dank seiner Größe und Lage eine Bundesrepublik im Kleinen. Die Kreise an der Küste im Norden des Landes profitieren vom Ausbau der Windkraft und produzieren Windstrom, den sie exportieren wollen. Wenn Hypothese 2.1 zutrifft, müssten hier parteipolitische Akteure zu finden sein, die dem Netzausbau positiv gegenüberstehen. Für sie ist beispielsweise die SüdLink Stromtrasse von großer Bedeutung, die Windstrom von der Nordseeküste in die süddeutschen Verbrauchszentren bringen soll. Die Landkreise im Süden des Landes dagegen haben kaum Windkraftkapazitäten. Sie sind aber Transitregionen. Qua ihrer geographischen Lage muss die SüdLink-Trasse bei ihnen vorbeikommen, ohne dass sie selbst davon profitieren. ${ }^{3}$

Innerhalb Niedersachsens haben wir sechs Landkreise aus dem Süden (Goslar, Göttingen, Hameln-Pyrmont, Hildesheim, Holzminden, Northeim) und aus dem Norden (Aurich, Cuxhaven, Emden, Friesland, Wesermarsch, Wittmund) ausgewählt. Abb. 1 zeigt, dass die nördlichen Landkreise viel Windstrom produzieren, die süd-

\footnotetext{
3 Selbst wenn diese Kreise Strom für den Export produzieren würden: Die SüdLink Trasse ist technisch so ausgelegt, dass eine Einspeisung vom Strom ,zwischendurch“ nicht möglich ist.
} 
lichen Landkreise dagegen nicht. ${ }^{4}$ Aber nicht nur die Windstromproduktion ist in den nördlichen Landkreisen stark, sie sind auch Standort wichtiger Unternehmen der Windenergiebranche, wie beispielsweise Enercon aus Aurich. ${ }^{5}$

Sollte Hypothese 2 zutreffen, müssten sich die lokalen Parteien aus den nördlichen Landkreisen für den Ausbau der Stromnetze positionieren, die lokalen Parteien aus den südlichen Landkreisen eher dagegen.

Aus Kenntnis der Konflikte des Politikfeldes (Fink und Ruffing 2019) kann eine Eingrenzung erfolgen, welche Themen von Parteien angesprochen werden könnten.

Die Nennung von zwei Themen deutet auf eine Position pro Netzausbau hin. Dies ist erstens die Beschleunigung des Netzausbaus, um die Energiewende nicht weiter zu verzögern. Zweitens ist dies der Verweis auf das ökonomische Potenzial der Windkraft für den Export und die Stromversorgung ganz Deutschlands.

Vier Themen dagegen signalisieren Positionen gegen den Netzausbau. Erstens kann die Windkraft auch als dezentrales Modell betont werden, demzufolge sie dazu dienen kann, den lokalen Strombedarf zu decken (wofür weniger Übertragungsnetze notwendig wären). Zweitens kann darauf hingewiesen werden, bei Entscheidungen über den Netzausbau stärker die Bürger*innen zu beteiligen. Drittens kann die Forderung geäußert werden, Stromtrassen als Erdkabel zu verlegen. Viertens können spezifische Trassen kritisiert werden, wie beispielsweise die für Niedersachsen wichtige Trasse SüdLink.

Der Zeitraum der Untersuchung ist von 2009 bis 2020. Im Jahr 2009 kam das Thema Stromnetzausbau durch das dritte Energiemarktpaket und den Erlass des EnLAG auf die Agenda der deutschen Politik. Ab 2009 konnte das Thema also auch potenziell auf die Agenda lokaler Parteien kommen (Fink et al. 2019, S. 147).

Die Auswahl der Parteien beschränkt sich auf CDU, SPD und Grüne. Bei diesen Parteien erwarten wir am wahrscheinlichsten eine Positionierung zum Netzausbau: CDU und SPD, weil sie als catch-all parties zu allen Politikfeldern eine Aussage machen müssen, bei den Grünen, weil Energie- und Umweltpolitik zu ihrem Markenkern gehört. Die vierte Partei, die im Untersuchungszeitraum durchgehend im Landtag vertreten ist, ist die $\mathrm{FDP}^{6}$ : Auch sie wäre ein interessanter Fall. In ihren Wahlprogrammen wendet sie sich einerseits gegen den Ausbau der Windenergie, andererseits befürwortet sie aber den Ausbau der Stromnetze. ${ }^{7}$ In der Summe sind aber

\footnotetext{
4 Siehe auch https://www.umwelt.niedersachsen.de/download/155141/Energiewendebericht_2019.pdf für eine Aufschlüsselung auf Gemeindebene.

5 Aurich galt zwischenzeitlich durch die Gewerbesteuereinnahmen durch Enercon als eine der reichsten Kleinstädte Deutschlands, https://www.deutschlandfunkkultur.de/flaute-in-der-windenergiebranchestellenabbau-bei-enercon.1001.de.html?dram:article_id=466356.

6 Die Linkspartei war 2008-2013 im Landtag, die AfD ist seit 2017 vertreten. Bei beiden Parteien tritt somit neben die generell lückenhafte Quellenlage auf der lokalen Ebene die geringe Zahl von Abgeordneten, über die sich Material finden ließe.

7 „Der ungezügelte Ausbau der Windenergie muss beendet werden. Der rot-grüne Windenergieerlass muss in seiner auf rücksichtslosen Ausbau zielenden Fassung wieder außer Kraft gesetzt werden.“ „Auch in Niedersachsen bestehen bereits Netzengpässe. Daher muss der Ausbau der Stromnetze und die Entwicklung technisch wirksamer sowie wirtschaftlich sinnvoller Stromspeichersysteme vorangetrieben werden." Beide Aussagen stammen aus dem Wahlprogramm der Freien Demokraten zur Landtagswahl in Niedersachsen 2017, https://landesverband-niedersachsen.stage.universum.com/sites/default/files/2019-11/FDPNiedersachsen-Landtagswahlprogramm-2017_0.pdf.
} 
von der FDP zu wenig lokale Parteiprogramme auffindbar, und auch die Zahl der Abgeordneten im Landtag ist zu gering, um dieses Defizit durch Rückgriff auf Aktivitäten der Abgeordneten auszugleichen (siehe unten für die empirische Strategie). ${ }^{8}$

Zur Erhebung der Parteipositionen wäre eine Kodierung von Wahlprogrammen ideal (Eder et al. 2017). Leider sind die Daten des Local Manifesto Projektes (LMP) nur für Großstädte vorhanden (Gross und Jankowski 2020b). Der Ausbau der Stromnetze betrifft aber ländliche Regionen, durch die Trassen führen, oder die viel Fläche haben, um Windstrom zu produzieren. Von den untersuchten Landkreisen hat nur die Stadt Göttingen mehr als 100.000 Einwohner und ist in den LMP Daten enthalten. Für die anderen Landkreise konnten nicht für alle Parteien Wahlprogramme gefunden werden (siehe den Anhang für eine Übersicht über das Material).

Der Artikel verfolgt daher eine zweischrittige Strategie, um eine empirische Sättigung zu erhalten. In einem ersten Schritt werden Wahlprogramme von Kreisverbänden aus den zwölf Kreisen manuell daraufhin ausgewertet, zu welchen Unterthemen des Stromnetzausbaus sie welche Positionen enthalten. ${ }^{9}$ Ergänzend dazu werden Pressemitteilungen der Kreisverbände ausgewertet.

In einem zweiten Schritt werden die Positionen der lokalen Landtagsabgeordneten ermittelt. Dieser Schritt ist begründungsbedürftig, könnten Landtagsabgeordnete doch auch als Repräsentanten ihrer Partei gesehen werden, denen wenig Spielraum bleibt, lokale Präferenzen zu verfolgen. Fruchtbarer ist es aber, Abgeordnete als Agenten mehrerer Prinzipale zu konzeptualisieren: Sie sind sowohl Agenten ihrer lokalen Wähler*innen als auch ihrer Parteien (Geese und Schwemmer 2019). Zwar herrscht bei den Abstimmungen Fraktionsdisziplin, aber bei ,weicheren“ Formen der Selbstpräsentation wie der Wahl der Ausschusszugehörigkeit (Manow 2012, S. 69-74) oder bei parlamentarischen Fragen (Zittel et al. 2019) können Abgeordnete von geographisch definierten Präferenzen geleitet werden. Als vote-seeking Akteure nehmen sie Präferenzen ihres Elektorates auf und signalisieren ihre Positionen. Daher dienen für unsere Untersuchung die Webseiten der Abgeordneten als Datengrundlage, ihre Pressemitteilungen, mit denen sie saliente Themen ihrer Agenda betonen können (Huber et al. 2020; Meyer und Wagner 2020), und außerdem ihre parlamentarische Aktivität in Form von Redebeiträgen und Anfragen.

Nicht alle Landtagsabgeordneten äußern sich zu Fragen des Netzausbaus und der Energiewende (siehe Tabelle A1 im Anhang). Dennoch finden sich ausreichend Abgeordnete sowohl in Nord- als auch in Südniedersachsen, die sich zum Netzausbau verorten (siehe Tabelle A2 im Anhang für eine Übersicht über das Material). Dabei zeigt sich ein kein klares Muster, dass sich an Mandatstypen oder der Regierung/ Opposition-Trennlinie festmachen ließe: Sowohl Abgeordnete der Regierungsparteien als auch der Opposition, sowohl Listenkandidat*innen als auch Direktkandidat*innen äußern sich.

\footnotetext{
8 Auch für die Linke und die AfD finden sich kaum Positionen, die Seiten der Kreisverbände sind oft nicht vorhanden oder schlecht gepflegt. Der Anhang legt unser gesamtes Datenmaterial offen, und zeigt die Lücken bei AfD, Linkspartei und FDP.

${ }^{9}$ Landkreise haben keine formellen Kompetenzen beim Stromnetzausbau, sie sind aber betroffen. Die Forschung zeigt, dass sich lokale Akteure auch zu Themen positionieren, zu denen sie keine Kompetenzen haben (Gross und Krauss 2019).
} 
In der Summe kann unser Design keinen vollkommen parallelisierten Vergleich leisten, der sich auf eine Sorte von Daten der Quelle stützt. Zu unterschiedlich ist das Kommunikationsverhalten der Abgeordneten und der Kreisverbände. Wir erreichen aber eine empirische Sättigung durch die Vielzahl an Material, die es uns erlaubt, ein Bild der Positionen lokaler Parteien und lokaler Repräsentant*innen zu gewinnen.

\section{Empirische Analyse}

Die empirische Analyse soll herausarbeiten, wie die Positionen der lokalen parteipolitischen Akteure zum Ausbau der Stromnetze sind. Dabei ist die Analyse nach Parteien - CDU, SPD, Grüne - gegliedert. Innerhalb der Parteien sind die nord- und südniedersächsischen Akteure kontrastiert.

Um Hypothese $1 \mathrm{zu}$ bewerten - die vermutet, dass die Position der lokalen Parteien der Position ihrer Landesverbände entspricht - ist es nötig, die Positionen der Landesverbände darzustellen. ${ }^{10}$ Die CDU Niedersachsen hat drei Positionen zum Netzausbau: Sie sieht erstens erneuerbare Energien als ,tragende Säule des Energiesystems “11, wofür zweitens ein Ausbau der Stromnetze nötig sei. ${ }^{12}$ Sie bevorzugt drittens die Erdverkabelung. ${ }^{13}$ Die SPD Niedersachsen artikuliert erstens die Notwendigkeit eines schnellen Netzausbaus: „Der Ausbau der Stromtrassen muss mit dem Ausbau der Erneuerbaren Energien Schritt halten. Nur dann kann die in Windkraftanlagen auf Land und See erzeugte Energie verlässlich genutzt werden. Deshalb bedeutet der Aufbau einer modernen und wettbewerbsfähigen Energieinfrastruktur eine große industrie- und umweltpolitische Chance für Niedersachsen. [...] Der Netzausbau dient zu einem grossen Teil der Versorgung anderer Bundesländer." 14 Sie nennt zweitens Transparenz und Bürgerbeteiligung bei der Planung als wichtige Ziele. ${ }^{15}$ Interessant ist drittens, dass die Forderung nach Erdkabeln im SPD-Wahlprogramm 2013 enthalten ist, 2017 aber nicht mehr. Die Grünen Niedersachsen versuchen ebenfalls, eine Abwägung in ihrem Parteiprogramm darzustellen. Sie sagen erstens ,Die Netzinfrastruktur darf kein Flaschenhals der Energiewende sein. ${ }^{16}$, setzen sich dabei aber zweitens für Erdkabel ein. ${ }^{17}$ Drittens bevorzugen sie dezentrale Stromproduktion ,,um möglichst viele Übertragungsnetz-

\footnotetext{
10 Für sich alleinstehend wirken alle folgenden Positionen sehr abgewogen und ,mittig“. Im Vergleich mit anderen Landesverbänden sind es aber Parteiprogramme, die den Netzausbau befürworten. Beispielsweise hatte die CDU Thüringen zu dieser Zeit in ihrem Programm „Wir wollen zusätzliche Stromtrassen durch Thüringen verhindern.".

11 CDU Niedersachsen, Wahlprogramm 2013.

12 CDU Niedersachsen Wahlprogramm 2017.

13 CDU Niedersachsen Wahlprogramm 2013 und 2017.

14 SPD Niedersachsen Wahlprogramm 2013.

15 SPD Niedersachsen Wahlprogramm 2013 und 2017.

16 Grüne Niedersachsen Wahlprogramm 2017.

17 Grüne Niedersachsen Wahlprogramm 2013 und 2017.
} 
trassen einzusparen“, und argumentieren viertens: „Mehr Akzeptanz gibt es nur durch intensive und frühzeitige Beteiligung der Bürger*innen“. ${ }^{18}$

Falls Hypothese 1 zutrifft, sollten sich die Positionen der Landesverbände auch in den lokalen Parteipositionen wiederfinden. Falls dagegen Hypothese 2 zutrifft, sollten lokale Akteure systematisch von den Positionen der Landesverbände abweichen.

Bei den $C D U$-Kreisverbänden in den nordniedersächsischen Landkreisen fällt zunächst auf, dass die kritischen Punkte zum Netzausbau nicht in Programmen und Pressemitteilungen vorkommen. Gerade das Fehlen der Forderung nach Erdverkabelung ist markant. Vielmehr wird die wirtschaftliche Bedeutung der Windenergie und ihr ökonomisches Potenzial für die Landkreise herausgestellt. Dies zeigt eine Pressemitteilug der CDU Aurich zur Kommunalwahl von 2016: „Auf Windkraftenergie als starken Wirtschaftsfaktor können und wollen wir nicht verzichten “. ${ }^{19}$ Auch die $C D U$ Wesermarsch sieht ,klimafreundlich erzeugten Strom [...] mittelfristig als Standortvorteil für energieintensive Betriebe im Landkreis““. ${ }^{20}$

Auch die Landtagsabgeordneten der CDU in den nordniedersächsischen Kreisen befürworten den Netzausbau. Bereits im Jahr 2012 sagte Björn Thümler, Abgeordneter des Kreises Wesermarsch - damals als Abgeordneter der Regierungskoalition: „Wenn es uns gelingt das enorme Potenzial der Offshore-Energie auszuschöpfen, wird auch die Umstellung auf eine ökologische Energieversorgung erfolgreich sein. Dafür muss beim Netzausbau dringend ein Gang hoch geschaltet werden“. ${ }^{21}$ Im Jahr 2016 saß Thümler mit der CDU in der Opposition und vertrat immer noch die selbe Position: Er übte Kritik an der rot-grünen Landesregierung hinsichtlich des zu langsamen Netzausbaus, Niedersachsen ,droht [...] seine Vorreiterrolle bei der Energiewende einzubüßen“. ${ }^{22}$ In Verbindung mit der Forderung nach einem schnelleren Netzausbau wird außerdem das wirtschaftliche Potenzial eines weiteren Ausbaus der Windenergie betont..$^{23,24}$

Die CDU-Kreisverbände in den südniedersächsischen Kreisen sprechen dagegen den Netzausbau kritisch an. Dies wird zwar nicht mit dem Versuch verbunden, sich pauschal gegen den Ausbau zu äußern, die lokalen Parteien beanspruchen aber für sich, die Sorgen der Bevölkerung zu artikulieren. Dies ist oftmals verbunden mit Kritik an der Vorgehensweise des Netzbetreibers TenneT. So meldet die CDU Northeim im März 2019 in einer Pressemitteilung dass ,,die Netzbetreiber selbst für ein Stocken des Netzausbaus [sorgen], indem sie den Weg des größten Widerstands

\footnotetext{
18 Grüne Niedersachsen Wahlprogramm 2017.

19 https://www.cduaurich.de/lokal_1_2_216_Pressemitteilung-zur-Kommunalwahl-2016.html.

20 https://www.cdu-wesermarsch.de/files/kreistagswahl_2016_wahlprogramm3.pdf.

$21 \mathrm{https} / / / \mathrm{cdu}$-niedersachsen.de/thumler-benotigen-beim-netzausbau-koordinierte-entscheidungen-aufbundesebene-niedersachsische-und-bremische-cdu-fraktionen-stellen-positionspapier-zur-windenergievor/.

22 https://www.cdu-fraktion-niedersachsen.de/presse/thuemler-rot-gruene-landesregierung-hatnetzausbau-verschlafen/.

23 https://celleheute.de/thumler-windenergie-bringt-neue-jobs-nach-niedersachsen.

24 https://www.thiemo-roehler.de/app/download/17039516396/Newsletter+November+2019.pdf?t= 1589390396.
} 
gehen." ${ }^{25}$ Gerade die kreispolitische Ebene dürfe dabei nicht die Verantwortung von sich weisen, sondern solle ,die Sorgen der Betroffenen im Blick haben und im Sinne des Schutzes von Mensch und Natur handeln und zusätzlichen Raumverbrauch möglichst verhindern“. Wenn Windenergie angesprochen wird, geht es weniger um den Export von Windstrom, sondern um die lokale Versorgung. Die CDU Göttingen betont daher: „Die Stadt und der Landkreis können dazu beitragen, dass [...] der Umstieg auf die lokale und dezentrale Energieversorgung gelingt. “26

Bei den CDU-Landtagsabgeordneten aus südniedersächsischen Landkreisen sind es vor allem Uwe Schünemann aus Holzminden sowie Otto Deppmeyer und Petra Joumaah aus Hameln-Pyrmont, die den Streckenverlaufsplan für SüdLink in ihren Wahlkreisen kritisieren. Sie richten als Oppositionspolitiker*innen Vorwürfe an die rot-grüne Landesregierung der Legislaturperiode 2013-2017, die sich nicht ausreichend in die Planung einbringe. Um den Genehmigungsprozess für die Stromtrassen zu beschleunigen, hatte die Bundesregierung den Landesbehörden die Kompetenzen für diesen Bereich entzogen. ${ }^{27}$ Der Landesregierung blieb somit lediglich die Option, einen nicht bindenden Vorschlag an die Bundesnetzagentur zu richten. Dazu forderte die CDU-Fraktion im Landtag sie auf. Schünemann bezieht sich hierbei in einer Pressemitteilung auf die Bedenken der Anwohner*innen im Landkreis. Diese seien ,zutiefst verunsichert und fordern zu Recht die Unterstützung der Landesregierung“. Diese drücke sich vor ihrer Verantwortung, für ein „transparentes Verfahren und die Offenlegung aller Kriterien zu sorgen" ${ }^{28}$. Ebenso betrachten auch Joumaah und Deppmeyer den geplanten Trassenverlauf durch ihren Wahlkreis als ungeeignet. "Warum das Landwirtschaftsministerium die Prüfung eines Trassenverlaufs in der Nähe von Bad Münder und Hameln anstrebt [...] bleibt vollkommen unklar.“29

Für die CDU zeigt sich, dass Kreistagsverbände und Landtagsabgeordneten - je nach geographischer Lage - eher die positiven oder die negativen Aspekte des Netzausbaus betonen.

Bei der SPD in den nordniedersächsischen Landkreisen ist die Datenlage schlecht. Es war lediglich möglich, das Kreiswahlprogramm der SPD Wesermarsch und der SPD Aurich von 2016 ausfindig zu machen. Außerdem bezieht sich nur die SPD Wesermarsch auf erneuerbare Energien im Allgemeinen und nicht auf den Netzausbau an sich. Nach der SPD Wesermarsch gehört die Windenergie zum Landkreis und der Ausbau und die Förderung regenerativer Energien soll verstärkt werden. ${ }^{30}$

Dafür finden sich Aussagen der SPD-Landtagsabgeordneten der nordniedersächsischen Kreise. Sie sind bezüglich des Netzausbaus zurückhaltender als die der $C D U$. Es müsse nach Olaf Lies, Abgeordneter des Kreises Friesland und seit 2017 niedersächsischer Umweltminister, ,Mit dem Ausbau der Windenergie [...] der Netz-

\footnotetext{
25 https://www.cdu-northeim.de/lokal_1_2_425_CDU-sieht-Suedlink-mit-Sorge.html.

26 Kreiswahlprogramm der CDU Göttingen 2011 (LMP).

27 Man beachte hier den Kontrast zu ihrem Parteifreund Björn Thümler aus Nordniedersachsen, der ebefalls von der Oppositionsbank aus der Landesregierung vorwirft, dass der Netzausbau zu langsam vonstatten gehe.

28 https://www.uwe-schuenemann.de/lokal_1_2_53_PRESSEMITTEILUNG--Suedlink.html.

29 https://www.weserbergland-nachrichten.de/hameln-pyrmont/6635-2015-02-26-07-53-40.

30 https://www.spd-wesermarsch.de/wp-content/uploads/sites/631/2018/06/Wahlprogramm.pdf.
} 
ausbau einhergehen“. ${ }^{31}$ Dabei bezeichnet er SüdLink als „eine wichtige Schlagader für die Versorgung der Republik mit erneuerbarer Energie“.32 Dennoch sieht er Niedersachsen überproportional vom Netzausbaumaßnahmen betroffen, daher müssten ,im niedersächsischen Verlauf weiterhin möglichst alle Belange berücksichtigt und Konflikte möglichst vermieden werden““.33,34 Neben Lies äußern sich keine anderen SPD-Landtagsabgeordneten zum Netzausbau. Im Mittelpunkt steht vielmehr der Ausbau der Windenergie zur Sicherung von Arbeitsplätzen und als Erfolgsfaktor der Energiewende. . $^{35,36}$

Ebenso wie bei der CDU finden sich bei den südniedersächsischen SPD-Verbänden deutlich mehr kritische Aussagen zum Netzausbau. In ihrem Programm zur Kommunalwahl 2016 befürwortet die SPD Göttingen den Ausbau erneuerbarer Energien und betont dabei die Anforderungen an Vereinbarkeit mit Natur- und Landschaftsschutz sowie die Bedürfnisse der Bevölkerung. ${ }^{37}$ Windstrom ist für diese Kreisverbände kein Exportgut. Wenn er angesprochen wird, geht es um die dezentrale Versorgung für den Eigenbedarf. Die SPD Göttingen ,[verfolgt daher] das Ziel der dezentralen Versorgung aus erneuerbaren Energien mit Augenmaß unter Beteiligung der Bürger*innen und Bürger". ${ }^{38}$ In Bezug auf SüdLink äußern die Kreisverbände der SPD ähnliche Kritik an TenneT wie die CDU - so bringt zum Beispiel die SPD Northeim in ihrem Wahlprogramm ihre grundsätzliche Akzeptanz für das Projekt zum Ausdruck, aber verbunden mit der Forderung nach einer vollständigen Erdverkabelung. „Im Schulterschluss mit den Bürgerinitiativen“ warnt die SPD, dass „wer erneut überflüssige Konflikte zu Gunsten der großen Stromversorger provoziert, auch mit der $380 \mathrm{KV}$-Leitung scheitern [wird]“. ${ }^{39}$ Auch die SPD Holzminden merkt 2011 in ihrem Wahlprogramm an, dass der Schutz des Landschaftsbildes Priorität habe und Stromkabel unter die Erde gehörten. ${ }^{40}$ Dieser Aspekt wird insbesondere von der SPD Hameln-Pyrmont aufgenommen. Ab 2014 strebte TenneT an, von der geplanten Strecke westwärts abzuweichen, wovon unter anderem das für den Tourismus bedeutsame Weserbergland in Hameln-Pyrmont und Holzminden betroffen

\footnotetext{
31 https://w3.windmesse.de/windenergie/pm/33831-land-niedersachsen-bundesland-rede-landtagumweltminister-olaf-lies-windkraftausbau-kritik-akzeptanz-spaltung-bevolkerung-anwohner-netzausbauklimawandel.

32 https://www.haz.de/Nachrichten/Der-Norden/Niedersachsen-Das-ist-der-aktuelle-Stand-bei-derSuedlink-Stromtrasse.

33 https://www.neuepresse.de/Hannover/Meine-Stadt/Suedlink-Trasse-soll-doch-an-Hannovervorbeifuehren.

34 https://www.nwzonline.de/wirtschaft/weser-ems/wilhelmshaven-hannover-netzausbau-inniedersachsen-das-sagt-minister-lies-zur-neuen-stromautobahn_a_50,5,2124367977.html.

35 https://www.cnv-medien.de/news/spd-landtagspolitiker-vorstoss-bei-offshore-wichtig-fuer-region. html.

36 https://www.matthias-arends.de/2019/11/19/matthias-arends-rueckenwind-fuer-onshore-wind/.

37 https://spd-ub-goettingen.de/uploads/ha_ub_goettingen/kreiswahlprogramm_2016_a4_download.pdf.

38 ebd.

39 Kreiswahlprogramm 2016 der SPD Northeim, Download unter https://spd-kreistagsfraktion-northeim. de/informationen.

40 https://spd-unterbezirk-holzminden.de/leitlinien-sozialdemokratischer-kreispolitik-2011-2016.
} 
war. ${ }^{41}$ Diese Änderung war für die Bürger*innen in Hameln-Pyrmont nicht akzeptabel und stieß dementsprechend auf Widerstand bei sämtlichen Parteien. Ein auf dem SPD-Unterbezirksparteitag 2015 beschlossener Antrag aus dem Kreisverband Hameln-Pyrmont lautet daher, dass die Trassenführung durch das Weserbergland ,absolut nicht nachvollziehbar sei““. ${ }^{42}$ Der Entwurf von TenneT sei zu unkonkret, lasse Fragen zu alternativen Trassenkorridoren offen und vernachlässige Umweltauswirkungen. Die lokalen Proteste führten zum Erfolg, als auf Bundesebene Ende 2015 der Beschluss zur priorisierten Erdverkabelung verabschiedet wurde. Knapp ein Jahr später revidierte TenneT zudem den „Westschwenk“. Die SPD Hameln-Pyrmont konstatiert daher in ihrem Wahlprogramm von 2016, ,dass sich gemeinsames Kämpfen gegen Eingriffe in den Naturpark Weserbergland lohnt" ${ }^{4}{ }^{43}$

Die Forderung nach Erdkabeln von fast allen südniedersächsischen SPD-Verbänden ist um so interessanter, als diese Forderung im Landtagswahlprogramm 2017 der SPD nicht mehr vorhanden ist. Dies ist einerseits erklärlich, weil die Möglichkeit der Erdverkabelung auf Bundesebene 2015 verankert wurde - die Landes-SPD muss dies also nicht mehr fordern. Andererseits ist es ein Thema, mit dem durch bloße Nennung Wähler*innenstimmen gewonnen werden könnten, und die Kreisverbände der SPD nutzen diese Forderung weiterhin, um mit Responsivität auf lokale Probleme Stimmen zu generieren.

Interessant gestaltet sich das Verhältnis zwischen den südniedersächsischen SPDLandtagsabgeordneten und der Landesregierung zwischen 2013 und 2017, zu deren Mehrheitsfraktionen die Abgeordneten gehörten. Diese stehen in einem Loyalitätskonflikt zwischen den Interessen ihrer Wähler*innen im Wahlkreis und denen der Landesregierung. Es findet sich wenig Material, welches auf eine konkrete Äußerung zu den Gegebenheiten im eigenen Wahlkreis in Opposition zu den Positionen der Landesregierung hindeutet. Ein Beispiel zeigt sich jedoch im Falle des $S P D$ Abgeordneten Ulrich Watermann aus Hameln-Pyrmont, der als Gemeinderatsmitglied in Bad Pyrmont 2014 eine parteiübergreifende Resolution des Rates gegen den Westschwenk durch das Weserbergland unterstützte und sein Unverständnis über diese Entscheidung bekundete. ${ }^{44}$ Hier ist die Dringlichkeit zu erkennen, mit der Parteipolitiker*innen im Kreis Hameln-Pyrmont unabhängig von der Position ihres Landesverbandes im Interesse der Wähler*innen eine Gefährdung ihrer Tourismusbranche verhindern wollen.

Auch bei den Grünen-Kreisverbänden in den nordniedersächsischen Landkreisen fällt auf, dass sie von den Positionen des Landesverbandes diejenigen betonen, die einen Ausbau der Stromnetze fordern, aber wenig zu den kritischen Aspekten sagen. Die Grünen Cuxhaven benennen die Notwendigkeit des Netzausbaus: ,[...] müssen

\footnotetext{
${ }^{41}$ Ein Grund für diese Entscheidung war mutmaßlich, dass Nordrhein-Westfalen, anders als die niedersächsische Landesregierung, keine Mindestabstände zu Wohngebieten für neue Stromtrassen vorgegeben hat.

42 https://spd-hameln-pyrmont.de/uploads/spdUnterbezirkHamelnPyrmont/antr_ge_und_ personalvorschl_ge_2015.pdf.

43 https://spd-hameln-pyrmont.de/unser-wahlprogramm-2016.

${ }^{44}$ https://www.dewezet.de/region/themendossiers/suedlink-dossier_artikel,-kein-westschwenk-durchsweserbergland-_arid,621052.html (Paywall).
} 
die landseitigen Stromnetze weiter modernisiert und ausgebaut werden". 45 Dagegen finden sich in den Programmen der Grünen häufig Verweise auf die ökonomischen Potenziale der Windkraft. So ist es den Grünen Wittmund und Wesermarsch ein Anliegen, bestehende Windkraftanlagen zu erneuern und aufzurüsten. ${ }^{46,47}$

Für die Landtagsabgeordneten der Grünen in Nordniedersachsen lassen sich keine Positionen bezüglich des Netzausbaus feststellen. Zum einen gibt es nur zwei grüne Abgeordnete in den nördlichen Wahlkreisen und zum anderen widmen sich diese dem Thema Netzausbau nicht. Eva Viehoff, Abgeordnete für den Wahlkreis Unterweser vertritt aber passend für ihre Region die Forderung nach einem Ausbau der Windenergie, um Arbeitsplätze zu erhalten und den Klimaschutz zu fördern. ${ }^{48}$

Die Grünen in den südniedersächsischen Landkreisen reihen sich mit ihren Forderungen in die Positionen der CDU und der SPD in Südniedersachsen ein. Auch bei ihnen steht Windenergie als dezentrale Stromversorgung im Vordergrund. Sollte es nicht anders möglich sein, unterstützen die Grünen auch den Ausbau der Übertragungsnetze. So sprechen sich die die Grünen Göttingen 2016 in ihrem Kreiswahlprogramm für dezentralen Windenergieausbau aus, verbunden mit einer Befürwortung des Netzausbaus an sich. ${ }^{49}$ Die Grünen Northeim und Hameln-Pyrmont setzen sich ebenfalls für dezentrale Energieerzeugung ein, ,,sollten dennoch Höchstspannungsleitungen benötigt werden, gehören sie unter die Erde“".50 Insbesondere der Verband im Kreis Holzminden hebt zusätzlich im Kreiswahlprogramm 2016 hervor, dass der Natur- und Artenschutz nicht durch den Stromnetzausbau gefährdet werden darf, welcher unter „,maximaler Bürgerbeteiligung“ durchzuführen sei. ${ }^{51}$ Auch die Kritik am Netzbetreiber TenneT setzt sich bei den Grünen fort und wird in Hameln-Pyrmont im Zusammenhang mit dem Westschwenk artikuliert: SüdLink „,bedarf Transparenz, Bürgerbeteiligung und eine nachhaltige Planung und [darf] nicht durch kurzfristige Gewinninteressen beeinflusst werden".52

Bei den südniedersächsischen grünen Landtagsabgeordneten ist interessant, dass sie im Untersuchungszeitraum Regierungsverantwortung hatten. Der Göttinger Abgeordnete Stefan Wenzel war 2013-2017 Landesumweltminister. Es ist folglich anzunehmen, dass seine Rolle als Landespolitiker überwiegt. So lehnte er 2013 eine Erdverkabelung der Stromtrasse Wahle-Mecklar trotz entsprechender Forderung von

\footnotetext{
45 https://www.cux-gruene.de/98_Umwelt__Energie.php?kurztext_ausklappen=374\#kurztext_98_ bausteine_3_374.

46 https://gruene-wittmund.de/wp-content/uploads/2016/09/Wahlgr\%c3\%b6nsnack2016-

LandkreisWittmund.pdf.

47 https://www.gruene-wesermarsch.de/userspace/NS/wesermarsch/KREISWAHLPROGRAMM-Gesamt2016.pdf.

48 https://www.eva-viehoff.de/presse/meldung/energiewende-blockade-muss-beendet-werden.html.

49 https://www.gruene-goettingen.de/userspace/NS/kv_goettingen/Homepage/KW-2016/LandkreisWahlprogramm_01.pdf.

50 https://www.gruene-northeim-einbeck.de/userspace/NS/northeim_einbeck/Kommunalwahlen_2016/ Kreistagswahlprogramm_2016.pdf.

51 Kreiswahlprogramm der Grünen Holzminden, zu finden unter https://www.gruene-holzminden.de/ home/.

52 https://www.gruene-hameln-pyrmont.de/wp-content/uploads/2014/12/PositionSuedlink_Gruene-HMP. pdf.
} 
Bürgerinitiativen ab. ${ }^{53}$ Der damalige Agrarminister Christian Meyer aus Holzminden setzte sich bereits seit 2011 für die Erdverkabelung ein. ${ }^{54}$ Auf den Antrag der CDUFraktion, die rot-grüne Landesregierung zur Formulierung eines eigenen Streckenverlaufsvorschlags für SüdLink aufzufordern, spielte Meyer den Ball an die Partei zurück: „Ich hoffe, dass Sie, liebe CDU, auch beim Bund dafür sorgen, dass es dort weiter Möglichkeiten für Teilerdverkabelung zum Schutz von Mensch und Umwelt gibt". 55

In der Summe ergibt sich daher ein aussagekräftiges Muster, das Themenschwerpunkte und -auslassungen zeigt (Tab. 1). Kaum einer der Kreisverbände und Abgeordneten nimmt die abwägenden Positionen der Landtagswahlprogramme ein. Stattdessen werden je nach Lage in Nord- oder Südniedersachsen die Punkte betont, die den Netzausbau unterstützen oder ihn kritisch sehen. Dabei ist von Bedeutung nicht nur was die lokalen Akteure sagen, sondern auch, welche Punkte sie nicht ansprechen (die instruktiven leeren Felder in der Tabelle). Die Parteien der nordniedersächsischen Kreise üben kaum Kritik am Ausbau der Stromnetze und erheben auch keine Forderung nach Erdverkabelung. Stattdessen betonen über alle drei Parteien hinweg die lokalen Vertreter*innen die wirtschaftliche Bedeutung der Windenergie als Exportgut. Dazu passt, dass Abgeordneten der nordniedersächsischen Kreise einen schnellen Netzausbau fordern.

Die Parteien in den südniedersächsischen Landkreisen äußern sich hingegen deutlich häufiger zu den kritischen Punkten des Stromnetzausbaus. Vor allem SPD und Grüne fordern Erdkabel. Außerdem findet sich hier über alle Parteien hinweg Kritik am Netzausbauprojekt SüdLink. Mit unterschiedlichen Schwerpunkten, aber insgesamt ähnlich, kritisieren $C D U$, SPD und Grüne das Vorgehen des Netzbetreibers TenneT, fordern die Erdverkabelung der Trasse und mehr Beteiligung der Bevölkerung. Die Grünen bekennen sich dabei am klarsten zur grundsätzlichen Unterstützung des Netzausbaus. Bei den Landtagsabgeordneten aus Südniedersachsen finden sich weniger Aussagen zum Netzausbau als von den Kreisverbänden. Die Abgeordneten der zu diesem Zeitpunkt oppositionellen $C D U$ nutzen ihr Mandat, um die Bedürfnisse der Anwohner*innen zu formulieren. Bei der SPD findet sich abgesehen von der Resolution des Rates der Stadt Bad Pyrmont, an der der Abgeordnete Watermann mitwirkte, kaum Material. Die Positionen der Grünen Abgeordneten aus Südniedersachsen entsprechen denen der Landesregierung, da sowohl Stephan Wenzel aus Göttingen als auch Christian Meyer aus Holzminden Ministerposten innehatten.

Zusammenfassend lässt sich daher H1 nicht bestätigen. Die Positionen der lokalen parteipolitischen Akteure sind kein Abbild der Positionen der Landesverbände. Auch H2 lässt sich in der formulierten Schärfe nicht bestätigen: Es gibt keine klare Trennung, derzufolge nordniedersächsische Parteien den Netzausbau befürworten, und südniedersächsische Parteien ihn ablehnen. Es zeigt sich aber eine Akzentuierung der Positionen: Über alle Parteien hinweg schweigen die nordniedersächsischen Parteien zu den kritischen Aspekten des Netzausbaus. Die südniedersächsischen

\footnotetext{
53 https://www.energate-messenger.de/news/137689/landesumweltminister-lobt-tennet (Paywall).

54 https://www.christian-meyer-gruene.de/presse/meldung/rede-christian-meyer-zur-akt-stunde-der-cduniedersachsen-macht-den-weg-frei-fuer-den-netzausbau.html.

55 https://www.landtag-niedersachsen.de/parlamentsdokumente/steno/17_wp/endber050.pdf (S. 4648).
} 


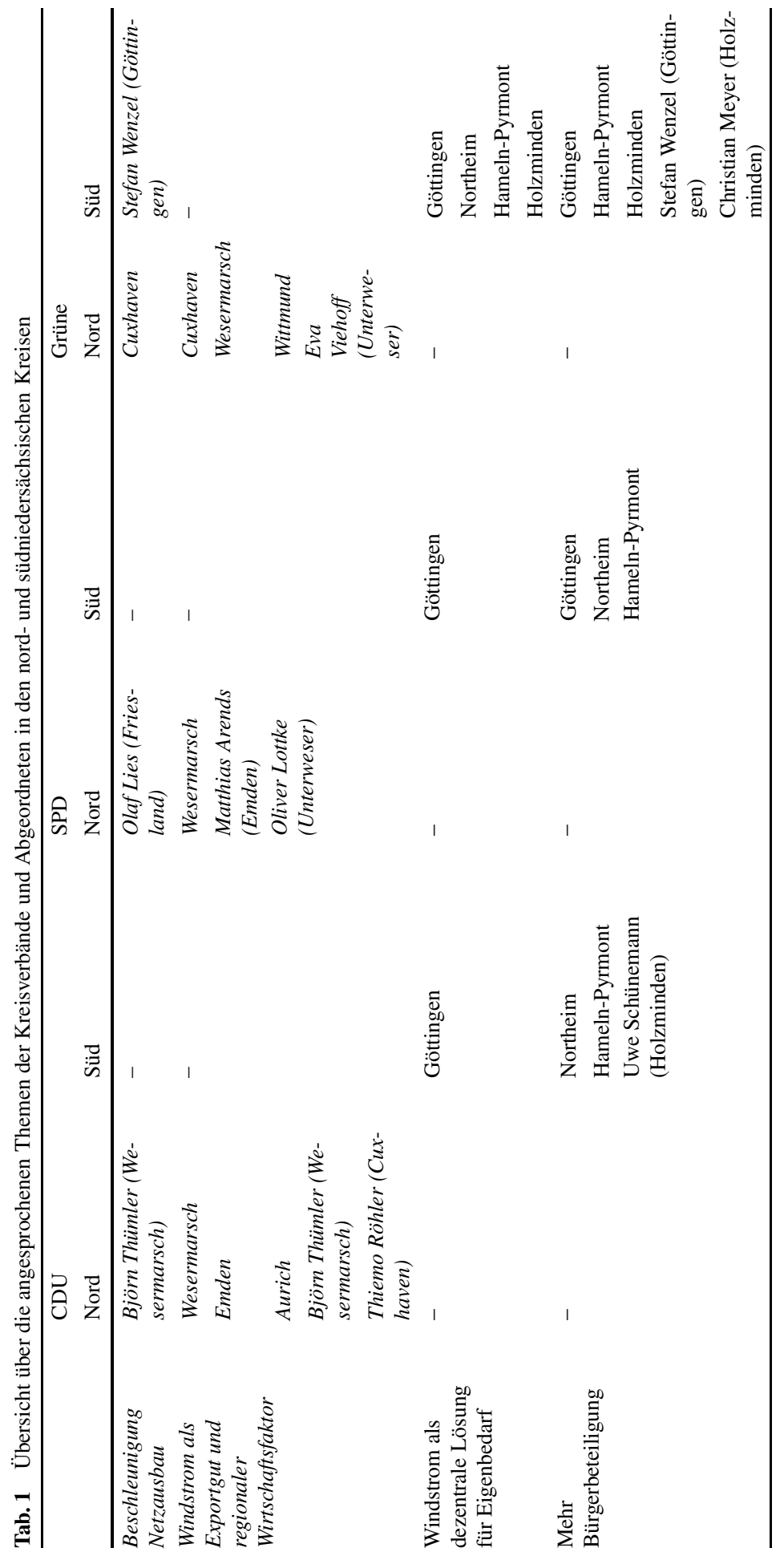




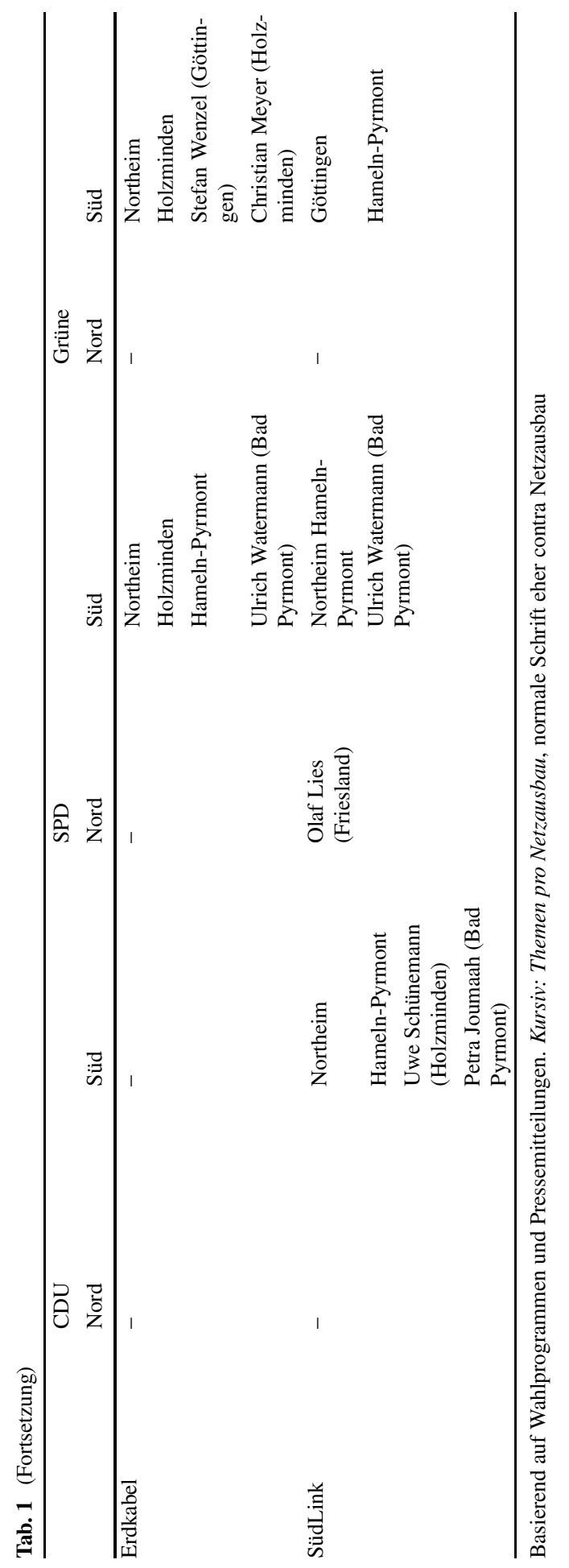


Parteien nennen zwar oft die Notwendigkeit des Netzausbaus, sie betonen aber die kritischen Aspekte. Außerdem stehen zwei unterschiedliche Ideen über die Rolle von Windstrom hinter den Positionen der lokalen Akteure: Für die Akteure aus Nordniedersachsen ist Windstrom ein Exportgut, das Stromtrassen nötig macht; für die Akteure aus Südniedersachsen ist Windstrom eine Möglichkeit der dezentralen Stromversorgung, die Stromtrassen überflüssig macht. In der Summe gibt es daher Evidenz für $\mathrm{H} 2.2$, weniger aber für $\mathrm{H} 2.1$.

\section{Zusammenfassung und Diskussion}

Der vorliegende Beitrag hatte zum Ziel herauszuarbeiten, ob lokale parteipolitische Akteure im Politikfeld Stromnetzausbau der Linie ihrer Landesverbände folgen (Hypothese 1), oder ob sie bedingt durch ihre energiepolitische Lage davon abweichen (Hypothese 2). Die Vermutung hinter Hypothese 2 war, dass es aus Gründen des vote seeking rational ist, eine kritische Position zum Stromnetzausbau einzunehmen, es sei denn, ein Landkreis profitiert vom Stromexport. Wir transformieren die übergeordnete Frage des special issue - die der Parteiendifferenz - in eine Frage der innerparteilichen Abweichung über die Ebenen des Mehrebenensystems hinweg.

Analysiert wurden die Hypothesen am Beispiel Niedersachsens. Niedersachsen hat aufgrund seiner Größe und Lage sowohl Landkreise, die vom Export von Windenergie profitieren, als auch Landkreise, die nur als Transitgegenden dienen.

In der Summe ergeben sich Belege für Hypothese 2, wenn auch nicht in der Klarheit, in der die Hypothese formuliert wurde. Es finden sich keine Kreisverbände, die der Linie ihrer Landesverbände offenkundig widersprechen. Die Kreisverbände betonen von den Positionen der Landesverbände aber jene, die ihrer energiepolitischen Lage entsprechen. Das Muster ist dabei trennscharf: Nur Landkreise im Süden Niedersachsens fordern Erdkabel und kritisieren die Trasse SüdLink, nur Landkreise im Norden Niedersachsens fordern eine Beschleunigung des Netzausbaus und nennen Windstrom als Exportfaktor.

Dieses Ergebnis trägt zu einigen Debatten bei, zeigt aber auch Lücken auf. Erstens zeigt das Ergebnis, dass der Stromnetzausbau in Deutschland unter einem Koordinationsproblem leidet. Auf Bundesebene herrscht Konsens über den Ausbau der Stromnetze, darunter liegen aber Konflikte zwischen den Bundesländern (Benz 2019; Ohlhorst et al. 2013), zwischen Landkreisen und Kommunen. Parteien als Mehrebenenorganisationen können oder wollen diese Konflikte nicht auflösen, sie spiegeln sie nur wider. Interessant wäre es, den umgekehrten Weg der Positionsentwicklung zu beleuchten: Wie kommen Bundesparteien oder Landesverbände zu Positionen, gegeben die große Heterogenität ihrer regionalen Einheiten?

Zweitens unterstützt unser Beitrag eine Sichtweise auf Parteien als lose gekoppelte Organisationen in denen territoriale Interessen eine Rolle spielen (Stecker 2015). Lokale Parteipositionen sind nicht Kopien der Bundesparteipositionen (Debus und Gross 2015, S. 836; Gross und Jankowski 2020a, S. 81). Im Politikfeld Netzausbau kann das durch die Spezifika des Politikfeldes - extrem unterschiedliche lokale Betroffenheit - begründet sein (Töller 2017). In diesem Sinne ist die Frage für das Politikfeld, inwieweit es Parteien gelingt, regional unterschiedliche Interessen 
- die durch Faktoren wie Not-in-my-backyard (NIMBY) Argumente bestimmt sind (Lindloff et al. 2017) - wieder zu integrieren.

Drittens ist unser Ergebnis für die Parteiendifferenztheorie eine Mahnung, Parteiendifferenz in Relation zur politischen Ebene zu sehen. Parteiendifferenzen auf Bundesebene sind nicht notwendigerweise auch auf Landes- oder regionaler Ebene vorhanden. Dies ist von Bedeutung, da in Mehrebenensystemen oft die Implementationskompetenz auf die Ebenen verteilt ist. Eine Parteiendifferenz auf Bundesebene kann bei der Implementation wieder aufgeweicht werden; umgekehrt bedeutet ein Fehlen von Parteienunterschieden auf Bundesebene nicht, dass keine Parteienunterschiede auf den implementierenden Ebenen bestehen. Parteiendifferenz ist immer abhängig von der betrachteten Ebene.

Zusatzmaterial online Zusätzliche Informationen sind in der Online-Version dieses Artikels (https://doi. org/10.1007/s12286-021-00494-5) enthalten.

Danksagung Eine frühere Version dieses Artikels wurde auf dem Workshop „Parteiendifferenz in der Umweltpolitik" vorgestellt. Wir danken den Teilnehmer*innen des Workshops sowie den Gutachter*innen für hilfreiche Hinweise.

Funding Open Access funding enabled and organized by Projekt DEAL.

Open Access Dieser Artikel wird unter der Creative Commons Namensnennung 4.0 International Lizenz veröffentlicht, welche die Nutzung, Vervielfältigung, Bearbeitung, Verbreitung und Wiedergabe in jeglichem Medium und Format erlaubt, sofern Sie den/die ursprünglichen Autor(en) und die Quelle ordnungsgemäß nennen, einen Link zur Creative Commons Lizenz beifügen und angeben, ob Änderungen vorgenommen wurden.

Die in diesem Artikel enthaltenen Bilder und sonstiges Drittmaterial unterliegen ebenfalls der genannten Creative Commons Lizenz, sofern sich aus der Abbildungslegende nichts anderes ergibt. Sofern das betreffende Material nicht unter der genannten Creative Commons Lizenz steht und die betreffende Handlung nicht nach gesetzlichen Vorschriften erlaubt ist, ist für die oben aufgeführten Weiterverwendungen des Materials die Einwilligung des jeweiligen Rechteinhabers einzuholen.

Weitere Details zur Lizenz entnehmen Sie bitte der Lizenzinformation auf http://creativecommons.org/ licenses/by/4.0/deed.de.

Interessenkonflikt S. Fink, H. Teichgräber und M. Wehling geben an, dass kein Interessenkonflikt besteht.

\section{Literatur}

Batel, S., und P. Devine-Wright. 2015. A critical and empirical analysis of the national-local „gap“ in public responses to large-scale energy infrastructures. Journal of Environmental Planning and Management 58:1076-1095.

Benz, Arthur. 2019. Koordination der Energiepolitik im deutschen Bundesstaat. dms - der moderne staat - Zeitschrift für Public Policy, Recht und Management 12:299-312.

Bräuninger, Thomas, und Marc Debus. 2012. Parteienwettbewerb in den deutschen Bundesländern, 1. Aufl., Wiesbaden: VS.

Carter, Neil. 2013. Greening the mainstream: party politics and the environment. Environmental Politics 22:73-94.

Däubler, Thomas, Jochen Müller, und C. Stecker. 2018. Assessing democratic representation in multi-level democracies. West European Politics 41:541-564.

Debus, Marc, und Martin Gross. 2015. Coalition formation at the local level: institutional constraints, party policy conflict, and office-seeking political parties. Party Politics 22:835-846. 
Debus, Marc, Christoph Knill, und Jale Tosun. 2012. Drum zahle, wer sich ewig bindet: Eine Analyse der Gebührenhöhe für eingetragene Lebenspartnerschaften in Baden-Württemberg. Politische Vierteljahresschrift 53:1-28.

Detterbeck, Klaus, und Wolfgang Renzsch. 2003. Multi-level electoral competition: the German case. European Urban and Regional Studies 10:257-269.

Eder, Nikolaus, Marcelo Jenny, und Wolfgang C. Müller. 2017. Manifesto functions: how party candidates view and use their party's central policy document. Electoral Studies 45:75-87.

Fink, Simon, und Felix Julian Koch. 2016. Agiert die Bundesnetzagentur beim Netzausbau als Agent oder als Treuhänder? dms - der moderne staat 9:277-288.

Fink, Simon, und Eva Ruffing. 2018. Öffentlichkeitsbeteiligung durch private Akteure: Eine empirische Analyse des deutschen Verfahrens zur Erstellung von Netzentwicklungsplänen. In Energiewende. Politikwissenschaftliche Perspektiven, Hrsg. Jörg Radtke, Norbert Kersting, 245-267. Wiesbaden: Springer.

Fink, Simon, und Eva Ruffing. 2019. Going beyond dyadic consultation relationships: information exchange in multi-step participation procedures. Journal of Public Policy 39:587-608.

Fink, Simon, Jan-Eric Bartels, Winona Hagendorf and Niklas Klinger. 2019. Determinants of subnational party positions on electricity grid expansion in Germany: Economic over political interests. Utilities Policy 58:145-157. https://doi.org/10.1016/j.jup.2019.05.007.

Funcke, Simon, und Dierk Bauknecht. 2016. Typology of centralised and decentralised visions for electricity infrastructure. Utilities Policy 40:67-74.

Geese, Lucas, und Carsten Schwemmer. 2019. MPs' principals and the substantive representation of disadvantaged immigrant groups. West European Politics 42:681-704.

Gross, Martin, und Michael Jankowski. 2020a. Dimensions of political conflict and party positions in multi-level democracies: evidence from the Local Manifesto Project. West European Politics 43:74-101.

Gross, Martin, und Michael Jankowski. 2020b. Lokale Wahlprogramme. Ein blinder Fleck der deutschen Kommunalpolitikforschung? In Neue Koalitionen - alte Probleme. Lokale Entscheidungsprozesse im Wandel, Hrsg. Björn Egner, Detlef Sack, 101-126. Wiesbaden: Springer VS.

Gross, Martin, und Svenja Krauss. 2019. Topic coverage of coalition agreements in multi-level settings: the case of Germany. German Politics. https://doi.org/10.1080/09644008.2019.1658077.

Holtkamp, Lars, Thomas Bathge, und Caroline Friedhoff. 2015. Kommunale Parteien und Wählergemeinschaften in Ost- und Westdeutschland. Zeitschrift für Vergleichende Politikwissenschaft 9:1-18.

Holtmann, Everhard, Christian Rademacher, und Marion Reiser. 2017. Kommunalpolitik : Eine Einführung. Wiesbaden: Springer VS.

Huber, Lena Maria, Anita Bodlos, Elisabeth Graf, und Thomas M. Meyer. 2020. Disseminating legislative debates: how legislators communicate the parliamentary agenda. Party Politics. https://doi.org/10. 1177/1354068820982555.

Kersting, Norbert, und Sebastian H. Schneider. 2016. Neue Machtansprüche in der Kommunalpolitik: Die Einstellungen von Ratsmitgliedern zu Bürgerbeteiligung. Zeitschrift für Vergleichende Politikwissenschaft 10:311-339.

Lindloff, Kirstin, Katharina Lisetska, Thomas Eisenmann, und Nils C. Bandelow. 2017. Regionale Betroffenheit in informellen Beteiligungsverfahren bei Infrastrukturprojekten. der moderne staat - dms: Zeitschrift für Public Policy, Recht und Management 10:89-115.

Manow, Philip. 2012. Wahlkreis-oder Listenabgeordneter, Typus oder Episode? Eine Sequenzanalyse der Wege in den Bundestag. Politische Vierteljahresschrift 53:53-78.

Messinger-Zimmer, Sören, und Julia Zilles. 2016. (De-)zentrale Energiewende und soziale Konflikte: Regionale Konflikte um die Vertretung des Gemeinwohls. Vierteljahreshefte zur Wirtschaftsforschung $85: 41-51$

Meyer, Thomas M., und Markus Wagner. 2020. Issue engagement across members of parliament: the role of issue specialization and party leadership. Legislative Studies Quarterly. https://doi.org/10.1111/ 1sq. 12308 .

Mueller, Christoph Emanuel, Silke Inga Keil, und Christian Bauer. 2019a. Effects of spatial proximity to proposed electric power lines on residents' expectations, attitudes, and protest behavior: A replication study. Energy Policy 130:341-346.

Mueller, Christoph Emanuel, Silke Inga Keil, und Christian Bauer. 2019b. Underground cables vs. overhead lines: quasi-experimental evidence for the effects on public risk expectations, attitudes, and protest behavior. Energy Policy 125:456-466.

Müller, Jochen. 2013. On a short leash? Sub-national party positions between regional context and national party unity. Journal of Elections, Public Opinion and Parties 23:177-199. 
Ohlhorst, Dörte, Kerstin Tews, und Miranda Schreurs. 2013. Energiewende als Herausforderung der Koordination im Mehrebenensystem. Technikfolgenanalyse. Theorie und Praxis 22:48-55.

Radtke, Jörg, und Norbert Kersting (Hrsg.). 2018. Energiewende. Wiesbaden: Springer VS.

Reusswig, Fritz, Florian Braun, Ines Heger, Thomas Ludewig, Eva Eichenauer, und Wiebke Lass. 2016. Against the wind: local opposition to the German Energiewende. Utilities Policy 41:214-227.

Schammann, Hannes. 2015. Wenn Variationen den Alltag bestimmen. Unterschiede lokaler Politikgestaltung in der Leistungsgewährung für Asylsuchende. Zeitschrift für Vergleichende Politikwissenschaft 9:161-182.

Schweizer-Ries, Petra. 2010. Abschlussbericht „Umweltpsychologische Untersuchung der Akzeptanz von Maßnahmen zur Netzintegration Erneuerbarer Energien in der Region Wahle-Mecklar (Niedersachsen und Hessen) “

Stecker, Christian. 2015. Parties on the chain of federalism: position-taking and multi-level party competition in Germany. West European Politics 38:1305-1325.

Steinbach, Armin (Hrsg.). 2013. NABEG, EnLAG, EnWG. Kommentar zum Recht des Energieleitungsbaus. Berlin: De Gruyter.

Töller, Annette Elisabeth. 2017. Verkehrte Welt? Parteien(in)differenz in der Umweltpolitik am Beispiel der Regulierung des Frackings. Zeitschrift für Politikwissenschaft 27:131-160.

ÜNB. 2017. Netzentwicklungsplan Strom 2030, Version 2017. Erster Entwurf der Übertragungsnetzbetreiber

Vogeler, Colette S., und Nils C. Bandelow. 2016. Devil Shift und Angel Shift in eskalierten politischen Konflikten am Beispiel von Stuttgart 21. Zeitschrift für Politikwissenschaft 26:301-324.

Zittel, Thomas, Dominic Nyhuis, und Markus Baumann. 2019. Geographic representation in party-dominated legislatures: a quantitative text analysis of parliamentary questions in the German Bundestag. Legislative Studies Quarterly 44:681-711. 